\title{
A Process for Assembling Discontinuous Basis Functions on Structured Hexahedral Grids
}

\author{
Ryan A. Chilton \\ AFRL, Wright-Patterson Air Force Base, Fairborn OH 45433
}

\section{Introduction}

Previous publications [1], [2] demonstrated conservative subgridding algorithms for structured grid methods (e.g. the Yee scheme, or the Lobatto Cell, a higher order extension [3]). These algorithms required a "nesting property" between the edge (facet) elements on a grid of size $\Delta L$ and the edge (facet) elements on a grid of size $\Delta L / 2$. Here, a general process for assembling basis functions is given, which does not require any nesting properties between the edge(facet) elements on adjacent grids. This development leads to very flexible spatial discretizations, where $h$ and $p$ can be picked on a cell-by-cell basis. The interfacing process is demonstrated on two 2D examples: (i) joining Lobatto Cell grids with equal $\Delta L$ but distinct polynomial order (non-uniform $p$-refinement), and (ii) subgridding the Yee scheme with an arbitrary $\mathrm{M}: \mathrm{N}$ ratio.

\section{Commutation Requirements for Conservative Interfaces}

Faradays and Amperes laws are traditionally expanded in space using edge elements for $\vec{E}$ and facet elements for $\vec{B}$, yielding the semi-discrete equations below (1), (2).

$$
\begin{aligned}
\frac{1}{c} \frac{\partial}{\partial t} \mathbf{b}(t) & =-\left(\mathbf{R}_{\mathbf{b}} \mathbf{R}_{\mathbf{b}}^{\mathrm{T}}\right)^{-1} \mathbf{R}_{\mathbf{b}} \mathbf{C R}_{\mathbf{e}}^{\mathrm{T}} \mathbf{e}(t) \\
\frac{1}{c} \frac{\partial}{\partial t} \mathbf{e}(t) & =\left(\mathbf{R}_{\mathbf{e}}\left[\star_{\epsilon}\right] \mathbf{R}_{\mathbf{e}}^{\mathrm{T}}\right)^{-1} \mathbf{R}_{\mathbf{e}} \mathbf{C}^{\mathrm{T}}\left[\star_{\mu^{-1}}\right] \mathbf{R}_{\mathbf{b}}^{\mathrm{T}} \mathbf{b}(t)
\end{aligned}
$$

Here, $\mathbf{C}$ is the curl stencil, $\left[\star_{\epsilon}\right]$ is a mass matrix of edge elements, and $\left[\star_{\mu^{-1}}\right]$ is a mass matrix of facet elements. The "restriction operators" $\mathbf{R}_{\mathbf{e}}$ and $\mathbf{R}_{\mathbf{b}}$ are used to assemble disjoint grids such that tangential field or normal flux representations are continuous across the boundary. Beyond $\mathbf{R}_{\mathbf{e}}$ and $\mathbf{R}_{\mathbf{b}}$, there are additional restriction operators for electric potential $\left(\mathbf{R}_{\phi}\right)$ and magnetic charge density $\left(\mathbf{R}_{\rho}\right)$. Collectively, these restriction operators should "commute" with the discrete differential stencils, $\mathbf{G}$ (gradient), $\mathbf{C}$ (curl) and divergence $\mathbf{D}$. This commutation is shown in (3) - (5).

$$
\begin{aligned}
& \mathbf{G R}_{\phi}^{\mathrm{T}}=\mathbf{R}_{\mathbf{e}}^{\mathrm{T}}\left[\left(\mathbf{R}_{\mathbf{e}} \mathbf{R}_{\mathbf{e}}^{\mathrm{T}}\right)^{-1} \mathbf{R}_{\mathbf{e}} \mathbf{G R}_{\phi}^{\mathrm{T}}\right]:=\mathbf{R}_{\mathbf{e}}^{\mathrm{T}}[\tilde{\mathbf{G}}] \\
& \mathbf{C R}_{\mathbf{e}}^{\mathrm{T}}=\mathbf{R}_{\mathbf{b}}^{\mathrm{T}}\left[\left(\mathbf{R}_{\mathbf{b}} \mathbf{R}_{\mathbf{b}}^{\mathrm{T}}\right)^{-1} \mathbf{R}_{\mathbf{b}} \mathbf{C R}_{\mathbf{e}}^{\mathrm{T}}\right]:=\mathbf{R}_{\mathbf{b}}^{\mathrm{T}}[\tilde{\mathbf{C}}] \\
& \mathbf{D R}_{\mathbf{b}}^{\mathrm{T}}=\mathbf{R}_{\rho}^{\mathrm{T}}\left[\left(\mathbf{R}_{\rho} \mathbf{R}_{\rho}^{\mathrm{T}}\right)^{-1} \mathbf{R}_{\rho} \mathbf{D} \mathbf{R}_{\mathbf{b}}^{\mathrm{T}}\right]:=\mathbf{R}_{\rho}^{\mathrm{T}}[\tilde{\mathbf{D}}]
\end{aligned}
$$

These requirements have two practical implications. First, the conjoined meshes support a new set of differential operators, $\tilde{\mathbf{G}}, \tilde{\mathbf{C}}, \tilde{\mathbf{D}}$, that exhibit the exact sequence property. This implies conservation of discrete electric and magnetic charge. Secondly, the spatially-discrete-time-continuous system (1), (2) is similar to an antisymmetric matrix. This implies that the scheme will be free of late-time instabilities when integrated with the leapfrog algorithm. 


\section{Building commutative restriction operators for tensor grids.}

The construction process is presented in 2D (Figure 1), although 3D considerations will follow. Assume that two conformal meshes, $M_{1}$ and $M_{2}$, are to be united at a common boundary which extends along $x$. Each mesh has its own set of nodal basis $[F]$ and segment basis $[G]$ functions which are used to represent field quantities at the shared interface.

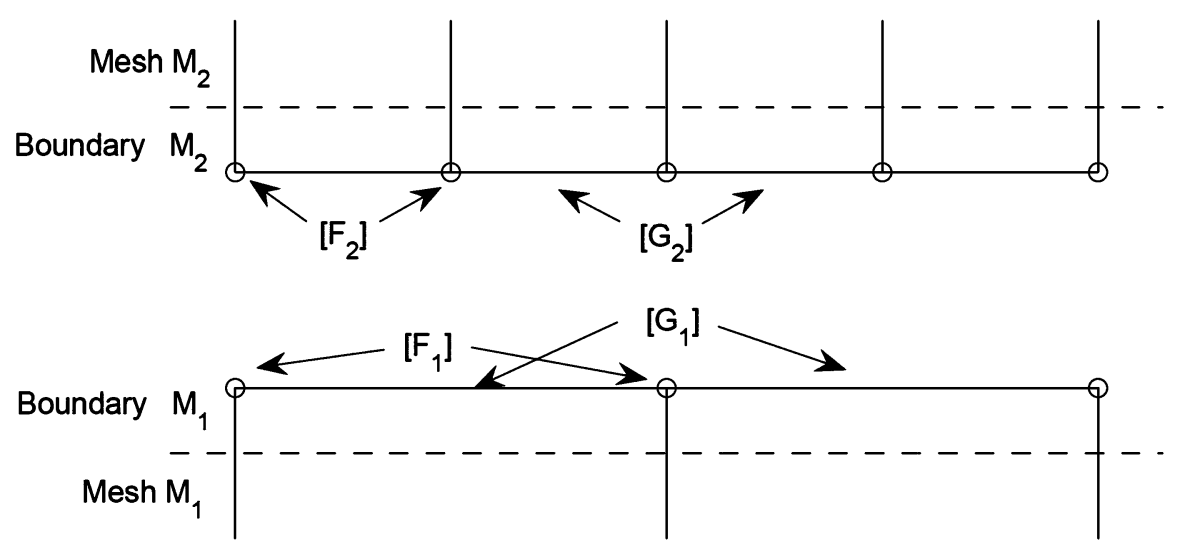

Figure 1: Assembling the $[F]$ and $[G]$ functions at a boundary shared between two meshes $M_{1}$ and $M_{2}$.

In the special case where the function spaces on $M_{1}$ and $M_{2}$ are nested, $\left[F_{1}\right]$ functions can be built from $\left[F_{2}\right]$ functions using a restriction operator $\mathbf{R}_{\mathbf{f}}$. The same can be said for the $[G]$ functions.

$$
\left[F_{1}\right]=\mathbf{R}_{\mathbf{f}}\left[F_{2}\right] \quad\left[G_{1}\right]=\mathbf{R}_{\mathbf{g}}\left[G_{2}\right]
$$

For any mixed order method, the derivative of any $f$-function can be represented exactly using a weighted sum of $g$-functions (like Yee's rooftops and pulses, for example). The one-dimensional partial derivative operator, $\mathbf{P}$, expresses this relationship. Each mesh boundary has its own distinct $\mathbf{P}$ operator.

$$
\frac{\partial}{\partial x}\left[F_{1}\right]=\mathbf{P}_{1}^{\mathrm{T}}\left[G_{1}\right] \quad \frac{\partial}{\partial x}\left[F_{2}\right]=\mathbf{P}_{2}^{\mathrm{T}}\left[G_{2}\right]
$$

Combining these relationships with the assembly operators of (6) reveals an interesting commutative property between one-dimensional differentiation and assembly.

$$
\mathbf{R}_{\mathbf{f}} \mathbf{P}_{2}^{\mathrm{T}}=\mathbf{P}_{1}^{\mathrm{T}} \mathbf{R}_{\mathbf{g}}
$$

A detailed consideration of the range and nullspaces of the $\mathbf{P}_{i}$ operators [4] reveals that this system is solvable if and only if all the columns of the $\mathbf{R}_{\mathbf{f}}$ operator sum to the same value, and it has linearly independent rows. Thus, a general design procedure for calculating assembly operators for arbitrary structured grid interfaces is sketched below. 
- Define the mesh with fewer nodes as $M_{1}$. This step guarantees that the $\mathbf{R}_{\mathbf{f}}$ operator has linearly independent rows.

- For each basis function $F_{i}$ supported on mesh $M_{1}$, evaluate it at every node location $j$ on mesh $M_{2}$. This sampled value is the coefficient $\mathbf{R}_{\mathbf{f}}[i, j]$. Because the $F_{i}$ 's are an interpolatory (partition of unity) basis, this step guarantees that each column of $\mathbf{R}_{\mathbf{f}}$ sums to a value of 1 .

- Compute $\mathbf{R}_{\mathbf{g}}=\left(\mathbf{P}_{\mathbf{1}} \mathbf{P}_{\mathbf{1}}^{\mathrm{T}}\right)^{-1} \mathbf{P}_{\mathbf{1}} \mathbf{R}_{\mathbf{f}} \mathbf{P}_{\mathbf{2}}{ }^{\mathrm{T}}$. This step solves $\mathbf{R}_{\mathbf{f}} \mathbf{P}_{\mathbf{2}}{ }^{\mathrm{T}}=\mathbf{P}_{\mathbf{1}}{ }^{\mathrm{T}} \mathbf{R}_{\mathbf{g}}$.

- In $2 \mathrm{D}, \mathbf{R}_{\phi}=\mathbf{R}_{\mathbf{f}}, \mathbf{R}_{\mathbf{e}}=\mathbf{R}_{\mathbf{g}}$ and $\mathbf{R}_{\mathbf{b}}=\mathbf{I}$. In $3 \mathrm{D}, \mathbf{R}_{\rho}=\mathbf{I}$ and the other operators are given by tensor products of $\mathbf{R}_{\mathbf{f}}$ and $\mathbf{R}_{\mathbf{g}}$ : A 0 -form is restricted using $\mathbf{R}_{\phi}=\mathbf{R}_{\mathbf{f}} \otimes \mathbf{R}_{\mathbf{f}}$, a 1-form is restricted using $\mathbf{R}_{\mathbf{e}}=\mathbf{R}_{\mathbf{f}} \otimes \mathbf{R}_{\mathbf{g}}$ and a 2-form is restricted using $\mathbf{R}_{\mathbf{b}}=\mathbf{R}_{\mathbf{g}} \otimes \mathbf{R}_{\mathbf{g}}$.

\section{M:N Subgridding for the Yee Scheme}

Figure 2 (left) shows example discretizations of a 1D boundary between two 2D Yee meshes. Both examples have $\mathrm{M}<\mathrm{N}$, so the size of the $\mathbf{R}_{\mathbf{f}}$ operator is $(M+1) \times(N+1)$. The entries of $\mathbf{R}_{\mathbf{f}}$ are calculated by sampling the coarser scalar nodal basis functions at the finer grid resolution. Assuming $\mathrm{M}$ and $\mathrm{N}$ are small, the $\mathbf{R}_{\mathbf{g}}$ operator can be computed in exact arithmetic, yielding rational stencil coefficients. Since the intcrfacc tiles pcriodically along $\mathrm{y}$, this small stcncil can bc applicd repcatcdly as needed.

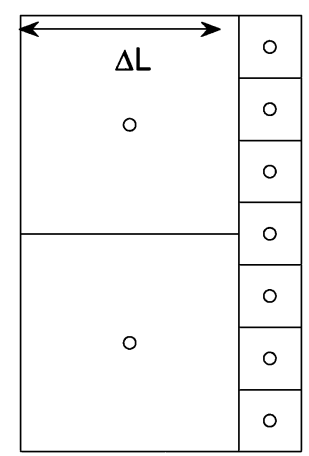

$M: N=2: 7$
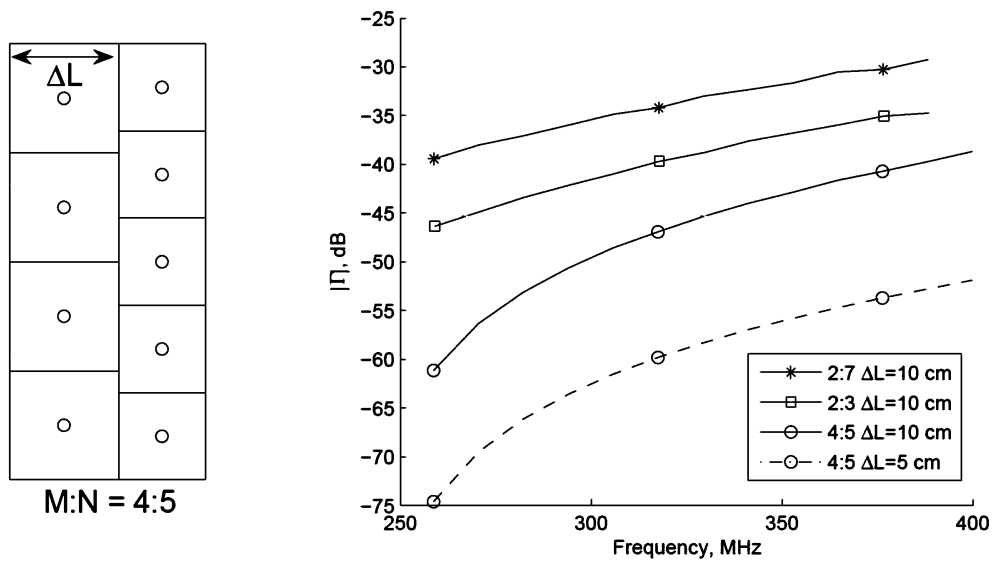

Figure 2: Spurious reflected field strength for Yee subgrids with arbitrary rational refinement ratios.

Reflection coefficients for various M:N ratios are reported in Figure 2 (right), for a parallel plate waveguide with $1.2 \mathrm{~m}$ separation. A $T E_{1}$ wave is launched from a grid with $\Delta L=10 \mathrm{~cm}$, which is then subdivided by assorted ratios. As expected from numerical dispersion considerations, the reflection coefficient shrinks as M:N approaches 1. An additional measurement, a 4:5 ratio with $\Delta L=5 \mathrm{~cm}$, indicates second order accuracy $\left(\left|\Gamma_{E}\right|\right.$ drops $12 \mathrm{~dB}$ when $\Delta L$ is halved). Though not supplied on the plot, the 2:3 and 2:7 cases also exhibit second order accuracy. 


\section{Non uniform P-refinement for the Lobatto Cell}

Here, the assembly process is applied to a non-uniform $p$-refinement problem: joining a Lobatto(2) grid to a Lobatto(3) grid at a 2D interface. See Fig. 3 (left).
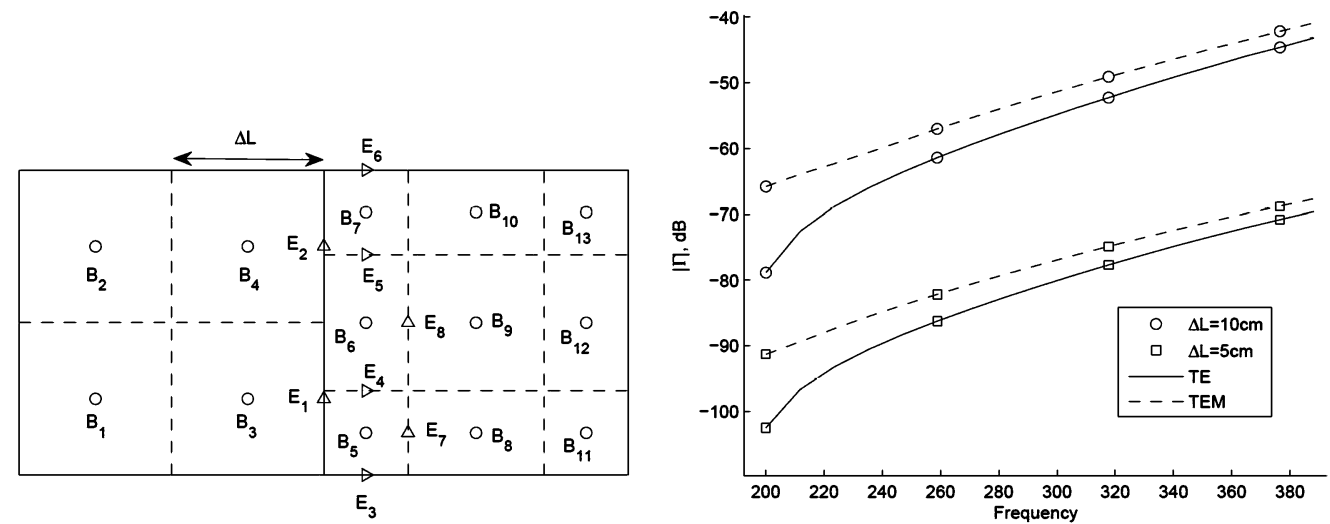

Figure 3: Spurious reflection coefficient measurement from interfacing Lobatto(2) and Lobatto(3) grids in a 2D waveguide.

Figure 3 (right) is a measurement of spurious reflection coefficient when a parallel plate waveguide mode propagates from a Lobatto(2) grid onto a Lobatto(3) grid. Both $T E_{1}$ and $T E M$ modes are tested, though the latter could also be measured on a 1D conformal mesh. For both excitations, the reflection coefficient converges to zero at a fourth order rate (halving $\Delta L$ results a $24 \mathrm{~dB}$ drop in $|\Gamma|$ ). This is intuitive, given the dispersion analysis within [3]. When the test is reversed and a wave propagates from the Lobatto(3) grid onto the Lobatto(2) grid, the results are identical.

\section{References}

[1] R. Chilton and R. Lee, "Conservative and Provably Stable FDTD Subgridding", IEEE-TAP, vol. 55, no. 9, pp. 2537-2549.

[2] R. Chilton and R. Lee, "Conservative Subgridding for Lobatto Cell FDTD Method,", AP Symposium, July 2008.

[3] R. Chilton and R. Lee, "The Lobatto Cell: Robust, Explicit, Higher Order FDTD that Handles Inhomogeneous Media", IEEE-TAP, vol. 56, no. 8, pp. 2167-2177.

[4] R. Chilton, "H-, P- and T- Refinement Strategies for the FDTD Method Developed via Finite-Element (FE) Principles", Ph.D. dissertation, 2008. 